\title{
Spectroscopic Investigation of Fluorescence Quenching Agents. Part III: Effect of Solvent Polarity on the Selectivity of Nitromethane for Discriminating Between Alternant Versus Nonalternant Polycyclic Aromatic Hydrocarbons
}

\author{
SHERYL A. TUCKER, WILLIAM E. ACREE, JR., ${ }^{*}$ JOHN C. FETZER, \\ RONALD G. HARVEY, MARY J. TANGA, PEI-CHAO CHENG, \\ and LAWRENCE T. SCOTT \\ Department of Chemistry, University of North Texas, Denton, Texas 76203-5068, (S.A.T., W.E.A.); Cheuron Research and \\ Technology Center, Richmond, California 94802-0627 (J.C.F.); Ben May Institute, University of Chicago, Chicago, Illinois \\ 60637 (R.G.H.); SRI International, 333 Ravenswood Avenue, Menlo Park, California 94025 (M.J.T.); and Department of \\ Chemistry, University of Nevada, Reno, Nevada 89557-0020 (P.-C.C., L.T.S.)
}

\begin{abstract}
To better assess the applicability of nitromethane as a selective quenching agent for alternant versus nonalternant polycyclic aromatic hydrocarbons in HPLC, TLC, and HPTLC analysis, we measured the effect that it has on the fluorescence emission behavior of 96 different polycyclic aromatic hydrocarbons dissolved in binary toluene/acetonitrile solvent mixtures. Results of these measurements revealed that the "selective quenching" rule is obeyed for the vast majority of PAHs, with the coronene derivatives being the only major exceptions. Fluorescence emission spectra are also reported for benzolg]chrysene, naphtho[2,3g|chrysene, $4 \mathrm{H}$-benzo[c]cyclopentalmno]chrysene, dibenzo[ghi,mno]fluoranthene (commonly called corannulene), rubicene, diacenaphtho[1,2j: $\left.\left.1^{\prime}, 2^{\prime}\right]\right]$ fluoranthene, 10-methylbenzo[b]fluoranthene, 3-methoxybenzo[k]fluoranthene, and 3-hydroxybenzo[k]fluoranthene in organic nonelectrolyte solvents of varying polarity. Calculated emission intensity ratios failed to vary systematically with solvent polarity, and all nine of the aforementioned solutes were thus classified as nonprobe molecules.

Index Headings: Fluorescence; Molecular structure; Spectroscopic techniques.
\end{abstract}

\section{INTRODUCTION}

Polycyclic aromatic compounds (PACs) have received considerable attention in the recent chemical literature, in part because of the widely varying carcinogenic and mutagenic properties of closely related isomers. Carcinogenic properties of select isomers, combined with an increasing awareness of environmental pollution and toxic material disposal, have prompted research to develop analytical methods specific for the different aromatic compounds. Current analytical methods generally employ high-resolution chromatographic techniques combined with pre-concentration via supercritical fluid ${ }^{1-5}$ or Soxhlet extraction, ${ }^{5-7}$ relatively sophisticated or lengthy isolation, or group separation schemes to obtain fractions from environmental or biological samples suitable for chromatographic analysis. Gas chromatographic separations utilizing liquid crystalline ${ }^{8-11}$ and/or polymeric liquid crystalline stationary phases, ${ }^{12-15}$ or using specially deactivated and stabilized thin-film open-tubular col-

Received 10 January 1993.

* Author to whom correspondence should be sent. umns, ${ }^{16,17}$ enable concentrations of several (though by no means all) of the aromatic constituents to be determined experimentally. Unfortunately, even these high-efficiency columns cannot completely resolve all polycyclic aromatic compound isomers found in environmental samples, and because of volatility constraints, quantification of the higher-molecular-weight PACs is not generally possible through gas chromatographic measurements.

Mixtures containing larger, nonvolatile polycyclic aromatic hydrocarbons can be conveniently analyzed by high-performance liquid chromatography (HPLC). Conventional chemically bonded monomeric $\mathrm{C}_{18}$ stationary phases $^{18-22}$ (i.e., phases prepared with monofunctional silanes) can generally separate the various PAH molecules present according to overall size and number of aromatic rings. Monomeric $\mathrm{C}_{18}$ phases possess only limited inherent ability to separate PAH isomers on the basis of molecular shape. Enhanced shape recognition is achieved on either a "charge transfer" 23 or a polymeric $\mathrm{C}_{18}$ stationary phase $\mathrm{e}^{18-22,24,25}$ (i.e., phase prepared with either difunctional or trifunctional silanes in the presence of water), the latter of which is believed to be molecularly ordered to some extent. Relative retention values for similar PAH structures of increasing size (i.e., naphthalene, pyrene, benzo[ghi]perylene, benzo[pqr]naphtho[ $8,1,2 \mathrm{bcd}]$ perylene, benzo[rst]dinaphtho[2,1,8,7defg; $2^{\prime}, 1^{\prime}, 8^{\prime} 7^{\prime} \mathrm{ijkl}$ )pentaphene, and tetrabenzo[def,lm,qrs,yz]pyranthene) with the use of different polymeric $\mathrm{C}_{18}$ stationary and mobile phase combinations, along with the elution strengths of the eleven common HPLC solvents, are reviewed in detail elsewhere. ${ }^{25}$

Conventional thin-layer chromatographic ${ }^{26-32}$ (TLC) and high-performance thin-layer chromatographic ${ }^{33-37}$ (HPTLC) methods have been employed with limited success in the analysis of unknown PAC samples. In many of the published studies, the various polycyclic aromatic compounds were separated on alumina, silica gel, 3-cyanopropylsilanized silica gel, 3-aminopropylsilanized silica gel, octadecylsilanized silica gel, or partially acetylated cellulose plates. The spots or zones corresponding to expected PACs were then excised, and the concentrations of the individual PAC isomers determined by elu- 
tion and solution fluorometry. As in the case of HPLC, resolution in thin-layer chromatography is deterrnined to a large extent by the stationary and mobile phase combination used. Even under optimum experimental conditions, it is very difficult (if not impossible) to completely separate all the different polycyclic aromatic compounds present in the environmental/biological samples commonly encountered.

Problems associated with co-eluting components in HPLC and TLC can be overcome in part by making the detector selective for each given PAC subclass. Of the four most common HPLC detectors on the market, fluorescence affords the most selectivity in that the excitation and emission wavelengths can be varied independently. While several polycyclic aromatic compounds may absorb at the same excitation wavelength, not all will emit at the wavelength(s) monitored by the detector. Utilization of selective fluorescence quenching agents further simplifies observed emission spectra by eliminating signals from undesired chemical interferences having only slightly different molecular structures. On the basis of limited fluorescence measurements for perylene, dibenzo[b,k]chrysene, dibenzo[h,rst]pentaphene, naphtho[ $1,2 \mathrm{~b}]$ fluoranthene, indeno[ $1,2,3 \mathrm{~cd}]$ pyrene, and 12,11-(peri-naphthylene)fluoranthene dissolved in a binary aqueous/acetonitrile mixture (20:80 percent by volume), Blümer and Zander ${ }^{38}$ noted that nitrobenzene and nitromethane selectively quenched fluorescence emission of the so-called "alternant" polycyclic aromatic hydrocarbons (PAHs). Emission intensities of the three nonalternant PAHs (e.g., naphtho[1,2b]fluoranthene, indeno[1,2,3cd]pyrene, and 12,11-(perinaphthylene)fluoranthene) were unaffected. Published studies ${ }^{39-44}$ involving over $63 \mathrm{PAHs}$ have identified dibenzo[hi,wx]heptacene, benzo[k]fluoranthene, and naphtho$[2,3 \mathrm{~b}]$ fluoranthene as among the few exceptions to the so-called nitromethane selective quenching rule in the PAH6 benzenoid, fluorenoid, fluoranthenoid, and "methylene-bridged" cyclopenta-PAH subclasses. More recent measurements ${ }^{45}$ revealed that nitromethane quenched fluorescence emission of all nine acenaphthylene-derivatives studied thus far, which is completely contrary to what would be expected on the basis of the fact that the nine solutes are nonalternant PAH molecules. Despite the aforementioned exceptions, nitromethane remains a very useful, selective quenching reagent in fluorescence analysis of unknown mixtures of polycyclic aromatic compounds.

For the most part previous nitromethane quenching investigations have been confined to more polar solvents such as neat acetonitrile or binary aqueous/acetonitrile mixtures. Both systems are used routinely as mobile phases in HPLC analysis of small PAHs having six rings or less. However, larger six- to ten-ring PAH molecules may require an aromatic hydrocarbon or a dichloromethane mobile phase cosolvent in order for the solutes to elute from the chromatographic column (or to dissolve from the TLC/HPTLC plate) in timely fashion. ${ }^{25}$ To better assess the applicability of nitromethane as a selective quenching agent for HPLC, TLC, and HPTLC analysis, we report the effect that nitromethane has on the fluorescence emission behavior of 96 different alternant and nonalternant PAHs dissolved in binary tolu-

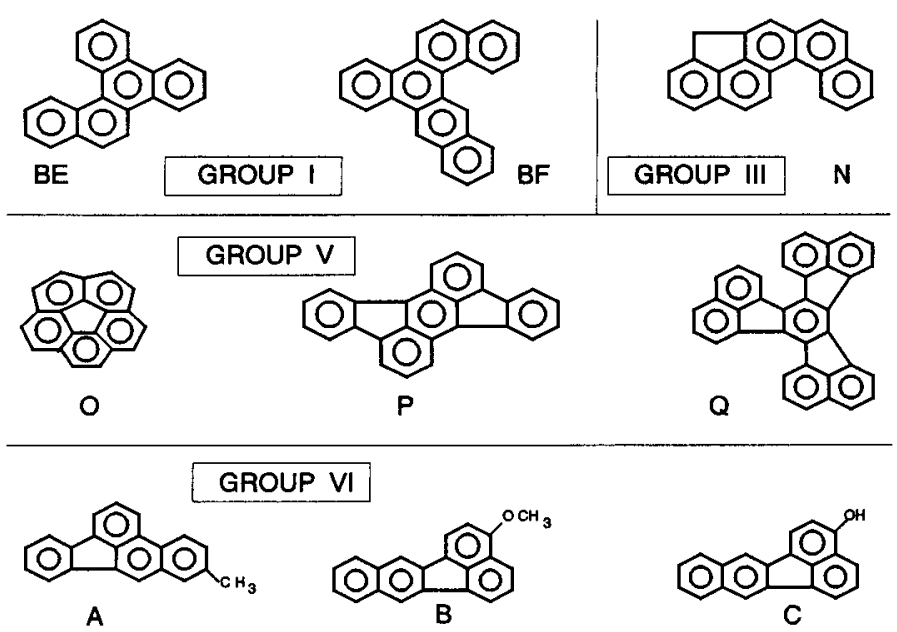

Fig. 1. Molecular structures of the polycyclic aromatic hydrocarbons: (BE) benzo[g]chrysene; (BF) naphtho[2,3g]chrysene; (N) $4 H$-benzo[c]cyclopenta[mno]chrysene; $(O)$ dibenzo[ghi,mno]fluoranthene (corannulene); (P) rubicene; $(\mathbf{Q})$ diacenaphtho[1,2j:1',2'l]fluoranthene; (A) 10-methylbenzo[b]fluoranthene; (B) 3-methoxybenzo[k]fluoranthene; (C) 3-hydroxybenzo[k]fluoranthene. See Table I for group definitions.

ene/acetonitrile solvent mixtures. Also, as a continuation of our fluorescence studies we have measured the fluorescence behavior of benzo[g]chrysene, naphtho[2,3g]chrysene, $4 H$-benzo[c]cyclopenta[mno]chrysene, dibenzo[ghi,mno]fluoranthene (commonly called corannulene), rubicene, diacenaphtho[ $\left[1,2 \mathrm{j}: 1^{\prime}, 2^{\prime} 1\right]$ fluoranthene, 10-methylbenzo[b] fluoranthene, 3-methoxybenzo[k]fluoranthene, and 3-hydroxybenzo[k]fluoranthene (see Fig. 1 for molecular structures) dissolved in nonelectrolyte organic solvents of varying solvent polarity.

\section{MATERIALS AND METHODS}

The nine new PAH solutes were synthesized and purified by procedures described in the chemical literature. ${ }^{46-53}$ 10-Methylbenzo[b]fluoranthene and 3-hydroxybenzo[k]fluoranthene are also available from the NCI Chemical Carcinogen Reference Standard Repository. ${ }^{54}$ Synthetical references and/or commercial suppliers for the remaining $\mathrm{PAH}$ solutes contained in Table I are listed in our earlier papers. Stock solutions were prepared by dissolving the solutes in dichloromethane. Small aliquots of the stock solutions were transferred into test tubes, allowed to evaporate, and diluted with the solvent of interest. Final solute concentrations were sufficiently dilute to minimize inner-filtering artifacts. Solvents were of HPLC, spectroquality or AR grade, purchased commercially from either Aldrich or Fisher Scientific, and the resulting solutions were optically dilute (absorbances $\mathrm{cm}^{-1}<0.01$ ) at all wavelengths, except for the nitromethane quenching studies.

Absorption spectra were recorded on a Bausch and Lomb Spectronic 2000 and a Hewlett-Packard 8450A photodiode array spectrophotometer in the usual manner. The fluorescence spectra were run on a Shimadzu RF-5000U spectrofluorometer with the detector set at high sensitivity. Solutions were excited at the wavelengths listed in Table I. Fluorescence data were accumulated in a $1-\mathrm{cm}^{2}$ quartz cuvette at $19^{\circ} \mathrm{C}$, ambient room 
TABLE I. Summary of excitation wavelength, probe character and fluorescence quenching (by nitromethane) data for alternant and nonalternant PAHs.

\begin{tabular}{|c|c|c|c|c|c|c|}
\hline Letter ${ }^{2}$ & Chemical name & Probe & $\begin{array}{c}\lambda_{e x} \\
(\mathrm{~nm})\end{array}$ & $\begin{array}{c}\lambda_{e m}^{\mathrm{b}} \\
(\mathrm{nm})\end{array}$ & $\begin{array}{c}\text { Quenched } \\
\text { ACN }\end{array}$ & $\begin{array}{c}\text { Quenched } \\
\text { ACN-TOLN }\end{array}$ \\
\hline \multicolumn{7}{|c|}{ Group I: Alternant PAHs } \\
\hline $\mathbf{A}$ & Coronene & Yes & 334 & 425 & Yes $^{d}$ & No \\
\hline $\mathbf{B}$ & Benzo[a]coronene & Yes & 316 & 429 & Yes $^{d}$ & No \\
\hline $\mathrm{C}$ & Naphtho[2,3a]coronene & Yes & 333 & 442 & Yes $^{d}$ & No \\
\hline D & Dibenzo[a,j]coronene & Yes & 324 & 431 & Yes $^{d}$ & No \\
\hline $\mathbf{E}$ & Naphtho[8,1,2abc]coronene & Yes & 322 & 458 & Yes $^{d}$ & No \\
\hline $\mathbf{F}$ & Naphtho $\left[2^{\prime}, 1^{\prime}, 8^{\prime}, 7^{\prime}: 4,10,5\right]$ anthra $[1,9,8 \mathrm{abcd}]$ coronene & No & 425 & & & \\
\hline $\mathbf{G}$ & Benzo $\left[1,2,3 \mathrm{bc} ; 4,5,6 \mathrm{~b}^{\prime} \mathrm{c}^{\prime}\right]$ dicoronene & No & 440 & & & \\
\hline $\mathbf{H}$ & Dibenzo[bc,ef]coronene & No & 366 & & & \\
\hline $\mathbf{I}$ & Tetrabenzo[de,hi,mn,qr]naphthacene & No & 318 & 418 & Yes & Yes \\
\hline $\mathbf{J}$ & Dinaphtho $\left[8,1,2 \mathrm{abc} ; 2^{\prime}, 1^{\prime}, 8^{\prime} \mathrm{klm}\right]$ coronene & Yes & 425 & & & \\
\hline $\mathbf{K}$ & Dinaphtho[8,1,2abc; $\left.2^{\prime}, 1^{\prime}, 8^{\prime} \mathrm{jkl}\right]$ coronene & $\mathrm{NA}^{\mathrm{c}}$ & 370 & 481 & Yes & No \\
\hline $\mathbf{L}$ & Anthra $[2,3 a]$ coronene & Yes & 357 & 488 & Yes $^{d}$ & No \\
\hline $\mathbf{M}$ & Pyrene & Yes & 338 & 371 & Yes & Yes \\
\hline $\mathbf{N}$ & Perylene & No & 403 & 437 & Yes & Yes \\
\hline $\mathbf{O}$ & Benzo[ghi]perylene & Yes & 380 & 405 & Yes & Yes \\
\hline $\mathbf{P}$ & Ovalene & Yes & 445 & & & \\
\hline $\mathbf{Q}$ & Naphtho[1,2,3,4ghi]perylene & No & 316 & 416 & Yes & Yes \\
\hline $\mathbf{R}$ & Benzo[pqr]naphtho $[8,1,2 \mathrm{bcd}]$ perylene & No & 325 & 418 & Yes $^{d}$ & Yes \\
\hline $\mathbf{S}$ & Dibenzo[cd,lm]perylene & No & 324 & & & \\
\hline $\mathbf{T}$ & Benzo[a]pyrene & No & 350 & 402 & Yes & Yes \\
\hline $\mathbf{U}$ & Benzo[e]pyrene & Yes & 335 & 386 & Yes & Yes \\
\hline $\mathbf{V}$ & Dibenzo[a,e]pyrene & No & 360 & 394 & Yes & Yes \\
\hline $\mathbf{W}$ & Dibenzo[fg,ij]phenanthro $[2,1,10,9,8,7$ pqrstuv]pentaphene & Yes & 393 & & & \\
\hline $\mathbf{X}$ & Tribenzo[de,kl,rst]pentaphene & No & 510 & & & \\
\hline $\mathbf{Y}$ & Dinaphtho[2,1,8,7defg; $2^{\prime}, 1^{\prime}, 8^{\prime}, 7^{\prime}$ ijkl]pentaphene & $\mathrm{NA}^{\mathrm{c}}$ & 420 & & & \\
\hline $\mathbf{Z}$ & Benz[rst]anthra[ $[9,1,2$ cde]pentaphene & $\mathrm{NA}^{\mathrm{c}}$ & 400 & & & \\
\hline $\mathbf{A A}$ & Benz $[4,10]$ anthra $[1,9,8$ abcd $]$ coronene & No & 370 & & & \\
\hline AB & Benzo[cd]chryseno $[4,5,6,7$ fghijk $]$ perylene & No & 330 & & & \\
\hline $\mathrm{AC}$ & Benzo[b]perylene & No & 312 & 437 & Yes $^{d}$ & Yes \\
\hline AD & Dibenzo[e,ghi]perylene & $\mathrm{NA}^{\mathrm{c}}$ & 330 & & & \\
\hline $\mathbf{A E}$ & Phenanthro $[5,4,3,2$ efghi $]$ perylene & Yes & 365 & & & \\
\hline AF & Dibenzo[cd,k]naphtho[ $8,1,2$ fghi]perylene & No & 321 & 451 & $\mathrm{Yes}^{\mathrm{d}}$ & Yes \\
\hline $\mathbf{A G}$ & Benzo[a]naphtho[ $8,1,2$ cde]naphthacene & No & 411 & 446 & Yes $^{d}$ & Yes \\
\hline AH & Dibenzo[def,p]chrysene & Yes & 310 & 419 & Yes & Yes \\
\hline AI & Anthranthrene & No & 306 & 430 & Yes & Yes \\
\hline AJ & Benzo[rst]pentaphene & Yes & 307 & 432 & Yes & Yes \\
\hline $\mathbf{A K}$ & Benzolrst]dinaphtho $\left[2,1,8,7 \mathrm{defg} ; 2^{\prime}, 1^{\prime}, 8^{\prime}, 7^{\prime} \mathrm{ijkl}\right]$ pentaphene & No & 315 & 438 & Yes & No \\
\hline $\mathbf{A L}$ & Dibenzo[de,kl]naphtho[ $[1,2,3,4$ rst $]$ pentaphene & No & 375 & & & \\
\hline $\mathbf{A M}$ & Dibenzo[h,rst]pentaphene & Yes & 366 & 416 & Yes $^{d}$ & Yes \\
\hline $\mathbf{A N}$ & Dibenzo[a,rst]naphtho[ $[8,1,2 \mathrm{cde}]$ pentaphene & No & 300 & 413 & Yes & Yes \\
\hline AO & Dibenzo[fg,ij]pentaphene & No & 300 & & & \\
\hline $\mathbf{A P}$ & Tetrabenzo[de,hi,op,st]pentacene & No & 375 & & & \\
\hline $\mathbf{A Q}$ & Dinaphtho $\left[2,1,8,7\right.$ defg; $; 2^{\prime}, 1^{\prime}, 8^{\prime}, 7^{\prime}$ opqr $]$ pentacene & No & 410 & 484 & Yes $^{d}$ & Yes \\
\hline $\mathbf{A R}$ & Pyranthene & $\mathrm{NA}^{\mathrm{c}}$ & 300 & 467 & Yes & Yes \\
\hline AS & Dibenzo[de,qr] naphthacene & Yes & 340 & & & \\
\hline AT & Dibenzo[de,st]pentacene & No & 408 & 470 & Yes & Yes \\
\hline $\mathbf{A U}$ & Dibenzo[fg,qr]pentacene & No & 400 & 435 & Yes & Yes \\
\hline AV & Naphtho[ $[8,1,2$ hij] hexaphene & No & 360 & 446 & Yes & Yes \\
\hline $\mathbf{A W}$ & Benzo[vwx]hexaphene & Yes & 360 & 477 & Yes & Yes \\
\hline $\mathbf{A X}$ & Benzo $\left[1,2,3 \mathrm{~cd} ; 4,5,6 \mathrm{c}^{\prime} \mathrm{d}^{\prime}\right]$ diperylene & No & 400 & 437 & Yes & Yes \\
\hline AY & Dibenzo[hi,wx]heptacene & No & 400 & 485 & No & No \\
\hline $\mathbf{A Z}$ & Benzo[b]triphenylene & No & 300 & 375 & Yes & Yes \\
\hline BA & Anthracene & No & 340 & 377 & Yes & Yes \\
\hline BB & Triphenylene & No & 300 & 354 & Yes & Yes \\
\hline $\mathbf{B C}$ & Phenanthrene & No & 300 & 363 & Yes & Yes \\
\hline BD & Chrysene & No & 320 & 380 & Yes & Yes \\
\hline BE & Benzolg]chrysene & No & 320 & 392 & Yes & Yes \\
\hline $\mathbf{B F}$ & Naphtho[2,3g]chrysene & No & 350 & 424 & Yes & Yes \\
\hline \multicolumn{7}{|c|}{ Group II: Alternant PAH derivatives } \\
\hline $\mathbf{A}$ & 1-Methylpyrene & No & 330 & 375 & $\mathrm{Yes}^{\mathrm{d}}$ & Yes \\
\hline B & 2-Methylpyrene & No & 333 & 374 & Yes $^{d}$ & Yes \\
\hline C & 4-Methylpyrene & No & 320 & 375 & Yes $^{d}$ & Yes \\
\hline $\mathbf{D}$ & 1,5-Dimethylpyrene & No & 320 & 379 & Yes $^{d}$ & Yes \\
\hline $\mathbf{E}$ & 1-Butylpyrene & No & 320 & 375 & Yes $^{d}$ & Yes \\
\hline $\mathbf{F}$ & 1-Decylpyrene & No & 320 & 375 & Yes $^{d}$ & Yes \\
\hline $\mathbf{G}$ & 4-Methylchrysene & No & 320 & 368 & Yes & Yes \\
\hline $\mathbf{H}$ & 5-Methylchrysene & No & 320 & 370 & Yes & Yes \\
\hline I & 5-Methyldibenzo[b,def]chrysene & No & 300 & 460 & Yes $^{d}$ & Yes \\
\hline $\mathbf{J}$ & 3,4,5-Trihydrobenzo[cd]pyrene & No & 340 & 377 & Yes & Yes \\
\hline
\end{tabular}


TABLE I. Continued.

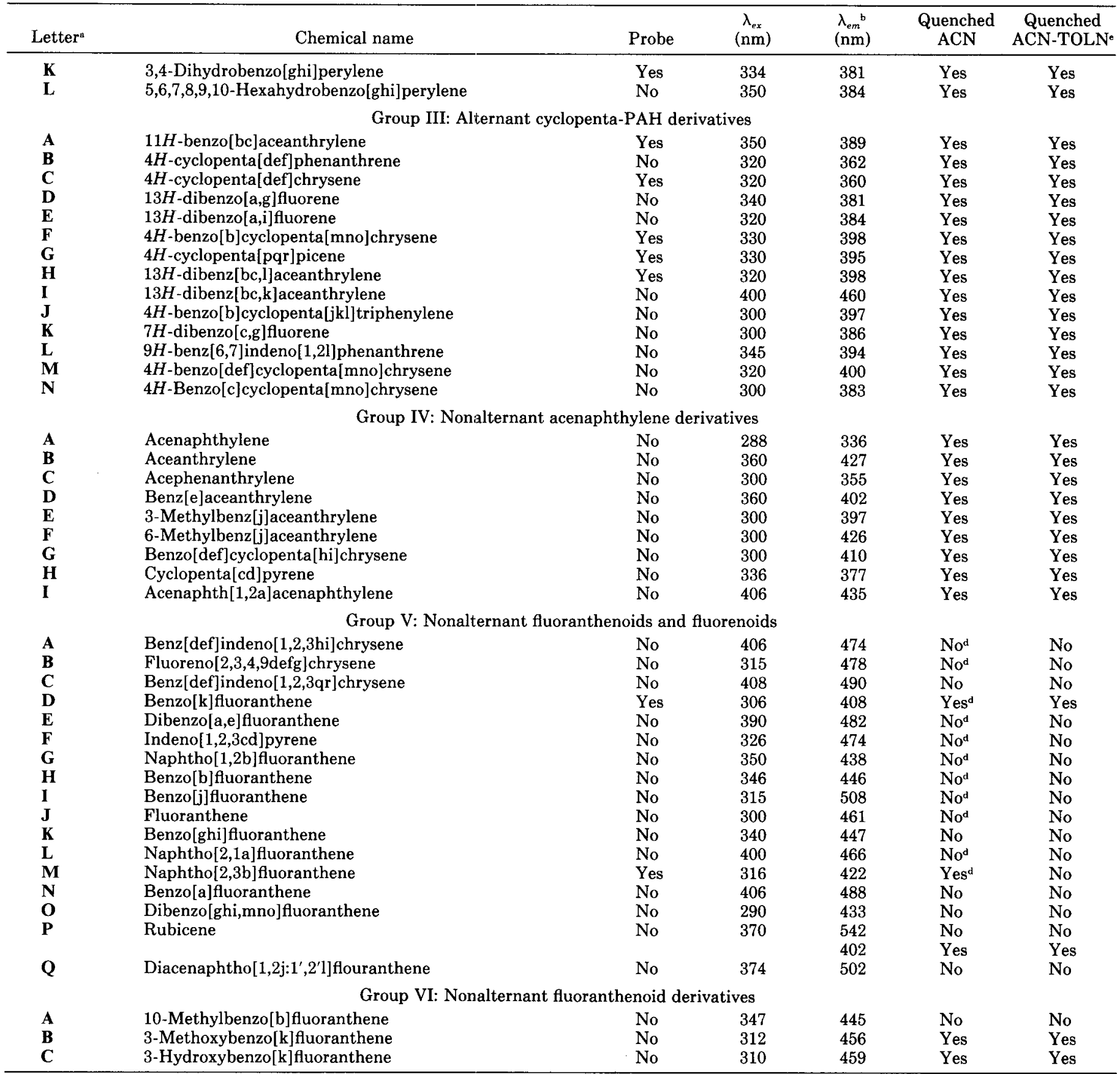

Molecular structures are given in Refs. 39-41, 45, 57, and 63.

${ }^{b}$ Emission wavelength used in the nitromethane quenching studies.

c NA indicates that the PAC is unacceptable as a solvent polarity probe molecule because a common set of bands could not be assigned in all solvents studied. The molecule may exhibit selective enhancement of one (or more) fluorescence emission band(s).

${ }^{d}$ Quenching studies performed in a binary aqueous/acetonitrile solvent mixture (20:80 percent by volume). Quenching of coronene and ben zo[a]coronene fluorescence emission, after correcting for primary inner-filtering and dilution, amounted to about a $20 \%$ reduction in signal intensity. Much larger reductions were observed for many of the other altenrant PAHs studied.

e Quenching studies performed in a binary acetonitrile/toluene solvent mixture (60:40 percent by volume).

temperature, with excitation and emission slit width settings of $15 \mathrm{~nm}$ and $3 \mathrm{~nm}$, respectively. For solvent polarity determinations involving pyrene, the emission slit width was reduced to $1.5 \mathrm{~nm}$ so that the calculated intensity ratios could be compared directly with literature values. ${ }^{55}$ Street and Acree ${ }^{56}$ had previously shown that emission intensity ratios vary with emission slit setting. The fluorescence spectra, depicted in Figs. 2 and 3, rep- resent a single scan which was then solvent blank corrected and verified by repetitive measurements.

Emission intensities associated with the quenching study were corrected for primary inner-filtering artifacts arising from the absorption of excitation radiation. Many of the PAHs have excitation wavelengths in the 300-320 $\mathrm{nm}$ spectral region, and a few drops of nitromethane gave solutions having appreciable absorbances. Mathematical 


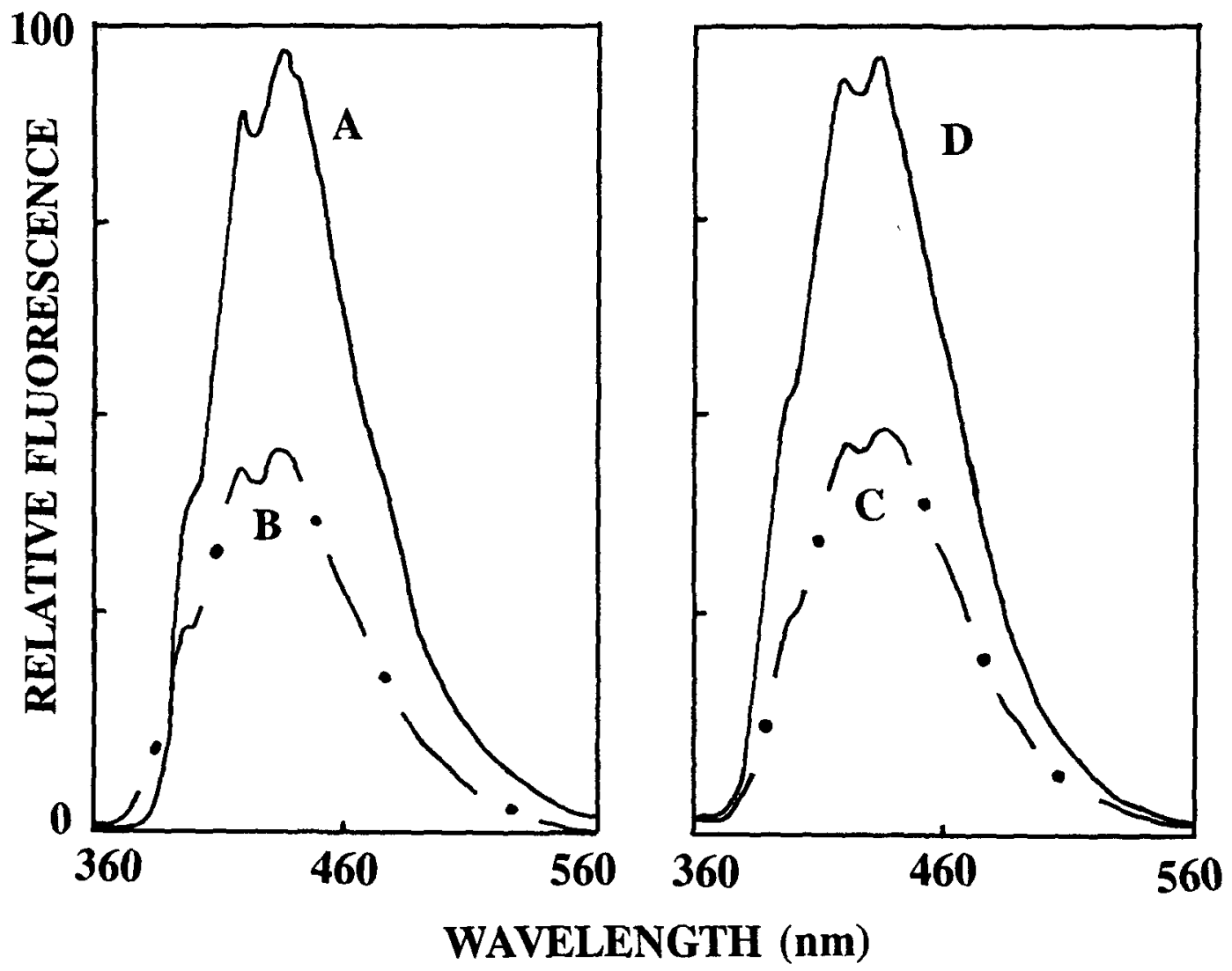

FIG. 2. Fluorescence emission spectra of corannulene dissolved in $[A(-)] n$-hexadecane; $[B(-\cdot-)]$ butyl acetate; $[C(-.-)]$ dichloromethane; and $[D(-)]$ dimethyl sulfoxide. In butyl acetate emission bands occur at about s-395, 419, and $434 \mathrm{~nm}$.

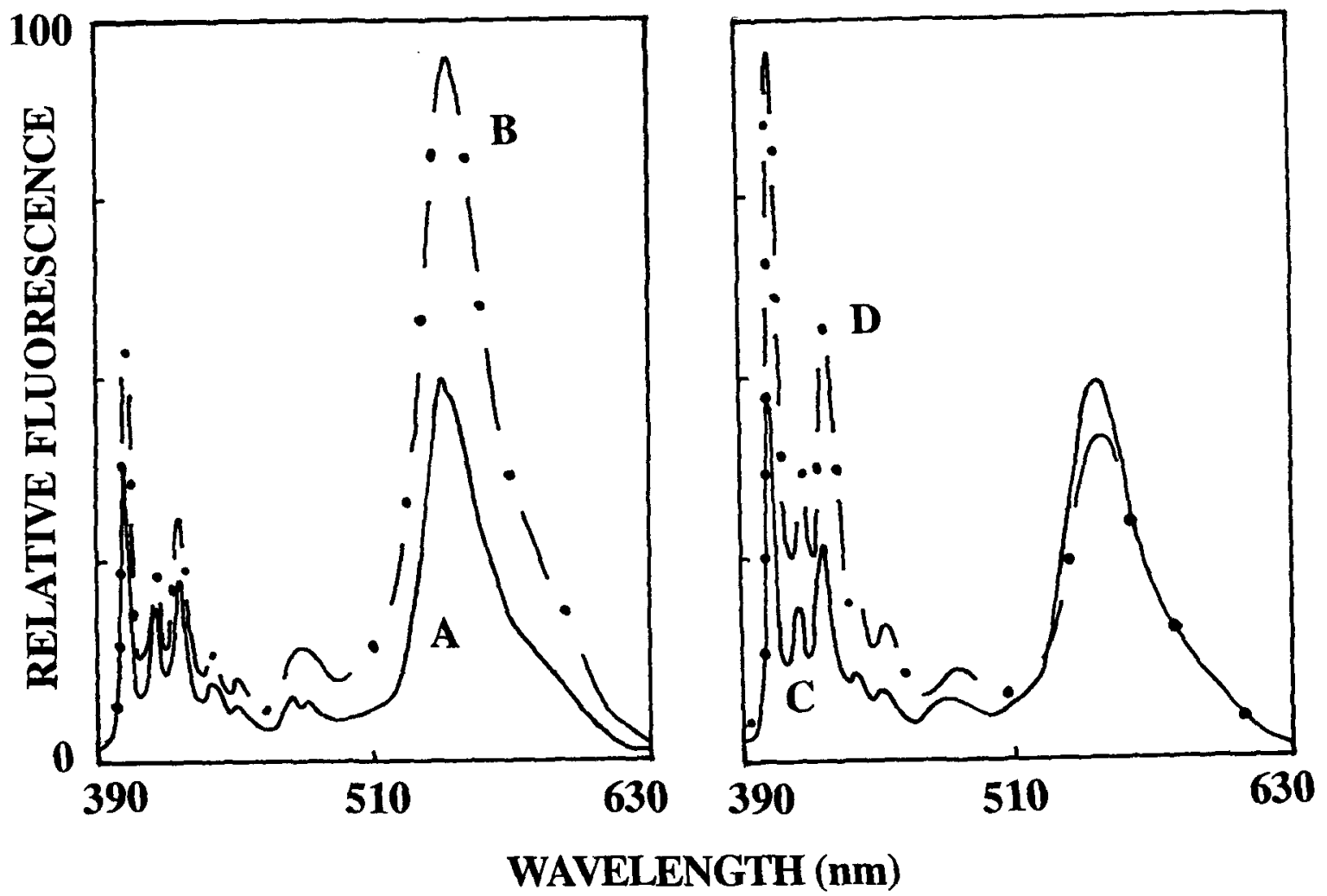

Fig. 3. Fluorescence emission spectra of rubicene dissolved in $[A(-)] n$-hexadecane; $[B(-.-)]$ butyl acetate; [C (-)] dichloromethane; and $[D(--)]$ dimethyl sulfoxide. In butyl acetate emission bands occur at about $402,415,425,439,450,478$, and $541 \mathrm{~nm}$. 
expressions, computational procedures, and interrogation zone dimensions are given elsewhere. ${ }^{39-41,57,53}$ Every effort was made to work at solution absorbances below $A \mathrm{~cm}^{-1} \leq 0.95\left(f_{\text {prim }} \leq 3.00\right)$ where the inner-filtering correction equation is valid. Secondary inner-filtering corrections were not necessary in the present study since nitromethane is "optically transparent" in most of these PAHs' emission ranges.

\section{RESULTS AND DISCUSSION}

Representative fluorescence emission spectra of corannulene (dibenzo[ghi,mno]fluoranthene) and rubicene dissolved in $n$-hexadecane, butyl acetate, dichloromethane, and dimethyl sulfoxide are depicted in Figs. 2 and 3. These four nonelectrolyte solvents were judiciously selected so as to encompass the entire range of solvent polarity, from the nonpolar $n$-hexadecane to the moderately polar butyl acetate and dichloromethane solvents to the very polar dimethyl sulfoxide, which is the most polar solvent considered in this present study. The fivemembered ring in the center of corannulene introduces curvature, giving it the shape of a bowl. The geometry of corannulene is reminiscent of the carbon cage molecules called fullerenes. Recent studies by Scott et al. suggest that its bowl inverts more than 200,000 times per second at room temperature. ${ }^{59}$ The fluorescence lifetime for most organic molecules is about $10^{-7}$ to $10^{-9}$ seconds; therefore, corannulene should not have time to complete a full inversion during the fluorophore's lifetime. The fluorescence spectra of corannulene in Fig. 2 are thought to be an average of the constantly inverting molecule. Although it is completely different in its molecular geometry than any other fluoranthenoid studied to date, its fluorescence emission spectra are typical for a member of this PAH subclass. Fluorescence emission spectra showed little or no fine structure and consisted primarily of 1-3 very broad bands for most of the fluoranthenoid and fluorenoid compounds studied.

Table II documents that within each group (see Table I for Group definitions) the newly studied PAHs' behavior is indicative of their respective subclasses, with the exception of rubicene. As with the previously studied pyrene derivatives, the substituted benzo[b]fluoranthene and benzo[k]fluoranthene fluorophores have fluorescence emission spectra similar to their parent compound, but with reduced fine structure. ${ }^{57}$ Closer examination of Fig. 3 reveals that rubicene initially showed signs of probe character as evidenced by variation of emission intensity ratios with solvent polarity. Calculated emission intensities for rubicene (RUB; I@ 403 nm/ VII@ $544 \mathrm{~nm}$ ) ranged from RUB $=0.80$ for $n$-hexadecane, RUB $=0.58$ butyl acetate, and $\mathrm{RUB}=0.96$ for dichloromethane to RUB $=2.16$ for dimethyl sulfoxide. The emission intensity ratios, however, do not vary systematically with solvent polarity regardless of how the emission peak ratios are defined. All of the solutes listed in Table II are thus classified as nonprobes. For a compound in the fluoranthenoid subclass, rubicene shows very unusual behavior. To date, we have not found any compound in this particular subclass to have more than three emission peaks in its fluorescence spectra. Rubicene has seven and sometimes eight emission peaks, and
TABLE 1I. Summary of excitation and emission wavelengths in butyl acetate for newly reported PAHs contained in Fig. 1.

\begin{tabular}{|c|c|c|c|}
\hline Letter & Chemical name & $\begin{array}{c}\lambda_{e x} \\
(\mathrm{~nm})\end{array}$ & $\begin{array}{c}\lambda_{e m} \\
(\mathrm{~nm})\end{array}$ \\
\hline \multicolumn{4}{|c|}{ Group I } \\
\hline $\mathbf{B E}$ & Benzo[g]chrysene & 320 & $379392 \mathrm{~s}-413^{\mathrm{a}}$ \\
\hline BF & Naphtho $[2,3 \mathrm{~g}]$ chrysene & 350 & 403422 \\
\hline \multicolumn{4}{|c|}{ Group III } \\
\hline $\mathbf{N}$ & $\begin{array}{l}4 H \text {-Benzo[c]cyclopenta[mno]- } \\
\text { chrysene }\end{array}$ & 300 & $384 \mathrm{~s}-397406429^{\mathrm{a}}$ \\
\hline \multicolumn{4}{|c|}{ Group V } \\
\hline $\mathbf{O}$ & Dibenzo[ghi,mno]fluoranthene & 290 & s-395 $419434^{\mathrm{a}}$ \\
\hline $\mathbf{P}$ & Rubicene & 370 & $\begin{array}{l}402415425439 \\
450478541\end{array}$ \\
\hline $\mathbf{Q}$ & $\begin{array}{l}\text { Diacenaphtho[ }\left[1,2 j: 1^{\prime}, 2^{\prime} 1\right]- \\
\text { fluoranthene }\end{array}$ & 374 & 474504 \\
\hline \multicolumn{4}{|c|}{ Group VI } \\
\hline $\mathbf{A}$ & $\begin{array}{l}\text { 10-Methylben- } \\
\text { zo[b]fluoranthene }\end{array}$ & 347 & 443 \\
\hline $\mathbf{B}$ & $\begin{array}{l}\text { 3-Methoxyben- } \\
\text { zo[k]fluoranthene }\end{array}$ & 312 & 430453 \\
\hline $\mathbf{C}$ & $\begin{array}{l}\text { 3-Hydroxyben- } \\
\text { zo[k]fluoranthene }\end{array}$ & 310 & 459 \\
\hline
\end{tabular}

a The s-denotes a shoulder.

most are very well resolved. In light of nitromethane quenching behavior (see below), the fluorescence characteristics of rubicene will be examined in greater detail.

Previous studies involving nitromethane as a selective quenching agent for discriminating between alternant vs. nonalternant PAHs utilized either neat acetonitrile or a binary aqueous/acetonitrile mixture (20:80 percent by volume). Such solvents work well as mobile phases in HPLC separations for those PAHs that contain six rings or less, but different solvent strengths must be employed for larger PAHs having six to ten rings. Toluene/acetonitrile or ethyl acetate/acetonitrile mixtures have served well in the past as HPLC mobile-phase solvents for the separation of the larger PAHs. ${ }^{25,60}$ Table I compares the experimental results regarding the ability of nitromethane to act as a selective quenching agent for alternant vs. nonalternant PAHs in the aqueous/acetonitrile mixture utilized earlier and a toluene/acetonitrile mixture (40:60 percent by volume). While a published paper ${ }^{38}$ has alluded to the fact that solvent polarity affects nitromethane's ability to act as a selective quenching agent, there was no in-depth study ever reported. Examination of Table I reveals that the toluene/acetonitrile mixture $(\mathrm{Py}=1.49)$, although less polar than the aqueous/acetonitrile mixture (observed $\mathrm{Py}=1.60^{61} ; \mathrm{Py}=1.80$ after correction for slit width effects ${ }^{56}$ ) or pure acetonitrile ( $\mathrm{Py}$ $=1.79^{55}$ ), can effectively be used as an HPLC solvent when nitromethane is employed to selectively quench the fluorescence emission signals of alternant vs. nonalternant PAHs. Of the 96 compounds examined, only nine behave differently in the toluene/acetonitrile solvent mixture as compared to the aqueous/acetonitrile solvent mixture or neat acetonitrile. Interestingly, seven of the nine exceptions are coronene derivatives. Earlier studies utilized the aqueous/acetonitrile mixtures, but we have found that for all 96 compounds, except for the aforementioned coronene derivatives, the selective quenching rule is obeyed in neat acetonitrile as well. Since then, we have employed acetonitrile, rather than the aqueous/ 


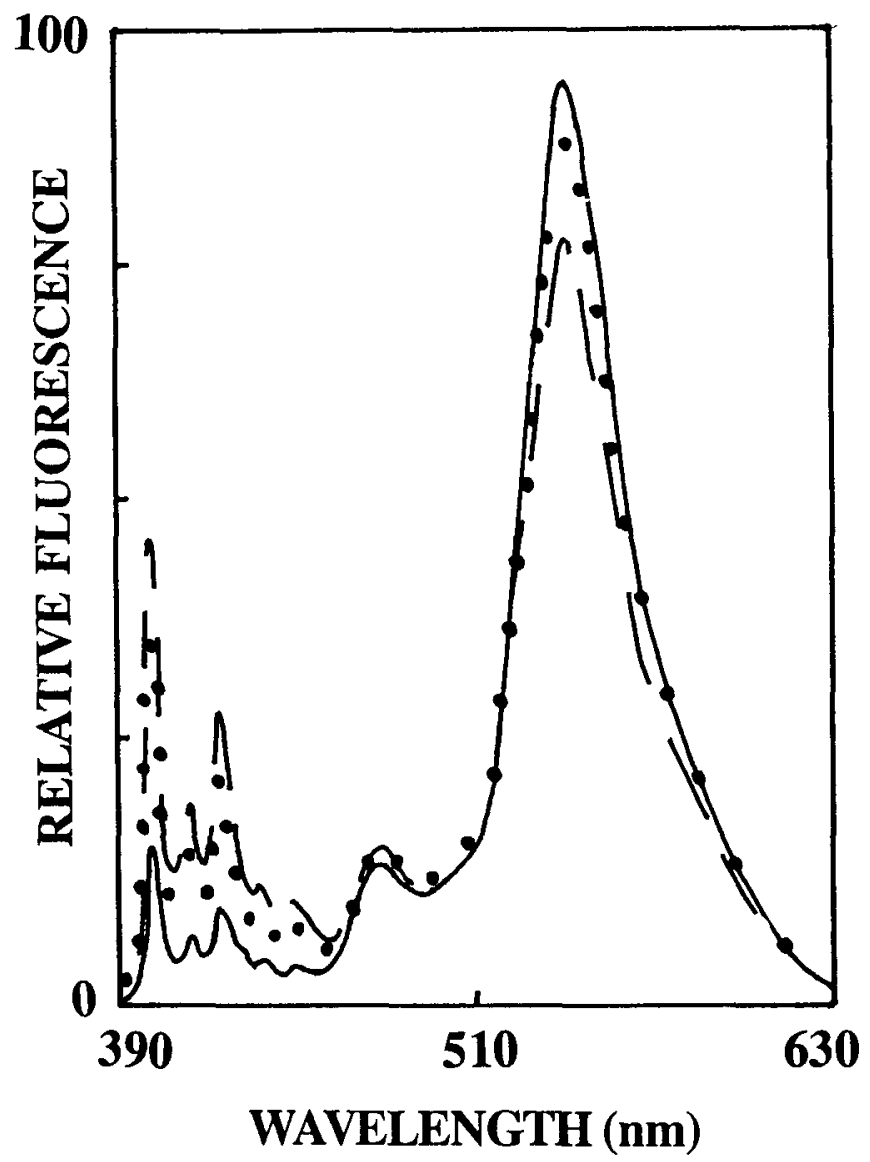

Fig. 4. Corrected fluorescence emission spectra of rubicene dissolved in acetonitrile at various nitromethane concentrations. From top to bottom in the $390-460 \mathrm{~nm}$ spectral range, the curves correspond to 0 , 1 , and 5 Pasteur pipet drops of nitromethane.

acetonitrile mixture, to enhance the solubility of the larger PAHs.

One of the unexpected "selective quenching" rule exceptions listed in Table $I$ is rubicene. Examination of Fig. 4 reveals that the quenching of rubicene is very different. Rubicene is a nonalternant fluoranthenoid; therefore, it should not be quenched by nitromethane. The first five emission peaks $(403,416,426,441$, and 453 $\mathrm{nm}$ ) that are narrow and well defined are quenched, while the two broad peaks (482 and $541 \mathrm{~nm}$ ) remain unquenched. The slight increase in intensity of the 541-nm emission peak via addition of nitromethane may be attributed to enhanced solubility, viscosity, or solvent polarity. As mentioned earlier, the number of peaks and the surprising amount of detailed fine structure of the first five peaks are not indicative of this subclass. The fluorescence emission spectra and the nitromethane quenching behavior suggest that rubicene acts as if it had two electronic centers, the first corresponding to an alternant moiety that would be quenched and the second corresponding to an unquenched nonalternant moiety. The molecular structure would also suggest this possibility since rubicene has an alternant anthracene moiety through the center of the compound as well as two nonalternant fluoranthene moieties on each end. The alternant moiety would explain the fine structure of the first five bands in the spectra, and the nonalternant moiety could explain the last two broad, unstructured bands.
Another plausible explanation of the broad red-shifted fluorescence emission peak at $541 \mathrm{~nm}$ in the rubicene spectra is excimer formation. To eliminate this possibility, we undertook a concentration study. It was found that even when the solute's concentration was so low that it gave only very weak fluorescence emission intensities, all seven or eight bands still remained, including the band at $541 \mathrm{~nm}$. The change in the excitation spectra via changing emission bands was also examined. In differing solvents the excitation spectra contained the same wavelengths at the various emission peak settings, but the ratios of the bands were significantly different. Also, the excitation spectrum with the emission collected at 541 $\mathrm{nm}$ was completely different than it was with the emission setting at $403 \mathrm{~nm}$. In dimethyl sulfoxide and $n$-hexadecane, the excitation spectra did not change when the emission setting was at any of five well-defined peaks (DMSO: 403, 416, 426, 441, and $453 \mathrm{~nm}$ ), but the excitation spectra were not the same as the spectrum obtained at $541 \mathrm{~nm}$. The small broad peak at $482 \mathrm{~nm}$ gave an excitation spectrum that appeared to be the addition of the two spectra obtained for the broad band at 541 $\mathrm{nm}$ and well-defined bands at 403, 416, 426, 441, or 453 $\mathrm{nm}$, since it was not identical to either one, but contained peaks belonging to both. Experimental data from the concentration and excitation studies, combined with the nitromethane quenching phenomena, as well as the molecular structure arguments, support the idea that rubicene acts like two electronic centers. A technique such as ultraviolet or fluorescence spectroscopy with polarized light in organized solvent media could perhaps determine whether this is true. ${ }^{62}$

Even in light of the few mentioned exceptions, nitromethane remains very useful as a selective quenching reagent. This work further provides a clear indication that it promises to be useful in actual HPLC separations. Future studies will examine several other HPLC solvents to verify that the "selective quenching" rule is obeyed.

\section{ACKNOWLEDGMENTS}

This work is supported in part by grants from the National Science Foundation (Grant No. CTS-8922485), by the University of North Texas Research Council, and by a National Science Foundation Doctoral Research Fellowship awarded to S. A. Tucker. The authors also acknowledge support from the National Institute of Environmental Health Sciences (Grant No. ES-04266), the National Cancer Institute (Contract No. N01-CP-15737), and the Department of Energy (Grant No. DE-FG08-88ER13945). They also thank Heather C. Bates and Vicki L. Amszi for making some of the preliminary measurements.

1. J. Rein, C. M. Cork, and K. G. Furton, J. Chromatogr. 545, 149 (1991).

2. B. W. Wright, S. R. Frye, D. G. McMinn, and R. D. Smith, Anal. Chem. 59, 640 (1987).

3. S. B. Hawthorne and D. J. Miller, J. Chromatogr. 403, 63 (1987).

4. S. B. Hawthorne, J. J. Langenfeld, D. J. Miller, and M. D. Burford, Anal. Chem. 64, 1614 (1992).

5. B. W. Wright, C. W. Wright, R. W. Gale, and R. D. Smith, Anal. Chem. 59, 38 (1987).

6. P. J. A. Fowlie and T. L. Bulman, Anal. Chem. 58, 721 (1986).

7. G. A. Junk and J. J. Richard, Anal. Chem. 58, 962 (1986).

8. G. M. Janini, K. Johnston, and W. L. Zielinski, Jr., Anal. Chem. 47, 670 (1975)

9. G. M. Janini, G. M. Muschik, and W. L. Zielinski, Jr., Anal. Chem. 48, 809 (1976).

10. W. L. Zielinski, Jr., R. A. Scanlan, and M. M. Miller, J. Chromatogr. 209, 87 (1981). 
11. J. E. Haky and G. M. Muschik, J. Chromatogr. 214, 161 (1981).

12. S. A. Wise, L. C. Sander, H. K. Chang, K. E. Markides, and M. L. Lee, Chromatographia 25, 473 (1988).

13. R. C. Kong, M. L. Lee, Y. Tominaga, R. Pratrap, M. Iwao, and R. N. Castle, Anal. Chem. 54, 1802 (1982).

14. M. A. Apfel, H. Finkelmann, G. M. Janini, R. J. Laub, B.-H. Lühmann, A. Price, W. L. Roberts, T. J. Shaw, and C. A. Smith, Anal. Chem. 57, 651 (1985).

15. K. E. Markides, M. Nishioka, B. J. Tarbet, J. S. Bradshaw, and M. L. Lee, Anal. Chem. 57, 1296 (1985).

16. M. L. Lee, F. J. Yang, and K. D. Bartle, Open Tubular Column Gas Chromatography: Theory and Practice (John Wiley and Sons, New York, 1984), pp. 249 and 312.

17. W. Bertsch, in Applications of Glass Capillary Gas Chromatography, W. G. Jennings, Ed. (Marcel Dekker, New York, 1981), p. 71.

18. S. A. Wise, L. C. Sander, R. Lapouyande, and P. Garrigues, J. Chromatogr. 514, 111 (1990)

19. L. C. Sander and S. A. Wise, Anal. Chem. 59, 2309 (1987).

20. L. C. Sander and S. A. Wise, J. High Resolut. Chromatogr. Chromatogr. Commun. 11, 383 (1988).

21. L. C. Sander and S. A. Wise, Anal. Chem. 61, 1749 (1989).

22. J. C. Fetzer, in Chemical Analysis of Polycyclic Aromatic Compounds, T. Vo-Dinh, Ed. (John Wiley and Sons, New York, 1989), Chap. 3.

23. L. C. Sander, R. M. Parris, S. A. Wise, and P. Garrigues, Anal. Chem. 63, 2589 (1991).

24. J. C. Fetzer and W. R. Biggs, J. Chromatogr. 386, 87 (1987).

25. J. C. Fetzer and W. R. Biggs, Chromatographia 27, 118 (1989).

26. S. K. Poole, T. A. Dean, and C. F. Poole, J. Chromatogr. 400, 323 (1987).

27. M. L. Lee, M. V. Novotny, and K. D. Bartle, Analytical Chemistry of Polycyclic Aromatic Compounds (Academic Press, New York, 1981).

28. R. J. Hurtubise, J. D. Phillip, and G. T. Skar, Anal. Chim. Acta 101, 333 (1978).

29. J. Kraft, A. Hartung, K.-H. Lies, and S. J. Schulze, J. High Resolut. Chromatogr. Chromatogr. Commun. 5, 489 (1982).

30. M. Katz and C. Chau, Environ. Sci. Technol. 14, 838 (1980).

31. A. H. Miguel, Int. J. Environ. Chem. 12, 17 (1982).

32. H. Matsushita, Y. Esumi, A. Suzuki, and T. Handa, Bunseki Kagaku 21, 1471 (1972).

33. C. F. Poole, H. T. Butler, M. E. Coddens, S. Khatib, and R. Vandervennet, J. Chromatogr. 302, 149 (1984).

34. W. A. Bruggeman, J, van der Steen, and O. Hutzinger, J. Chromatogr. 238, 335 (1982).

35. B. Seifert, J. Chromatogr. 131, 417 (1977).

36. S. S. J. Ho, H. T. Butler, and C. F. Poole, J. Chromatogr. 281, 330 (1983).

37. H. T. Butler, M. E. Coddens, S. Khatib, and C. F. Poole, J. Chromatogr. Sci. 23, 200 (1985).
38. G.-P. Blümer and M. Zander, Fresenius Z. Anal. Chem. 296, 409 (1979).

39. S. A. Tucker, W. E. Acree, Jr., B. P. Cho, R. G. Harvey, and J. C. Fetzer, Appl. Spectrosc. 45, 1699 (1991).

40. V. L. Amszi, Y. Cordero, B. Smith, S. A. Tucker, W. E. Acree, Jr., C. Yang, E. Abu-Shaqara, and R. G. Harvey, Appl. Spectrosc. 46, 1156 (1991).

41. S. A. Tucker, H. Darmodjo, W. E. Acree, Jr., J. C. Fetzer, and M. Zander, Appl. Spectrosc. 46, 1260 (1992).

42. H. Dreeskamp, E. Koch, and M. Zander, Z. Naturforsch. 30A, 1311 (1975).

43. U. Breymann, H. Dreeskamp, E. Koch, and M. Zander, Chem. Phys. Lett. 59, 68 (1978).

44. S. H. Chen, C. E. Evans, and V. L. McGuffin, Anal. Chim. Acta 246, 65 (1991)

45. S. A. Tucker, H. C. Bates, V. L. Amszi, W. E. Acree, Jr., H. Lee, P. D. Raddo, R. G. Harvey, J. C. Fetzer and G. Dyker. Anal. Chim. Acta, paper in press.

46. L. T. Scott, M. M. Hashemi, D. T. Meyer, and H. B. Warren, J. Am. Chem. Soc. 113, 7082 (1991).

47. S. Amin, K. Huic, N. Hussain, G. Balanijkas, S. G. Carmella, and S. S. Hecht, J. Org. Chem. 51, 1206 (1986).

48. M. J. Tanga and J. E. Bupp, J. Org. Chem., submitted for publication.

49. K. Dziewonski and Z. Leyko, Ber Deutschen Chem. Gesell. 47, 1679 (1914).

50. A. Schönberg and K. H. Brosowski, Chem. Ber. 93, 2149 (1960).

51. A. Schönberg and K. H. Brosowski, Chem. Ber. 95, 1910 (1962).

52. C. Yang, D. T. C. Yang, and R. G. Harvey, Synlett 799 (1992).

53. R. G. Harvey, C. Yang, and H. Lee, manuscript in preparation.

54. NCI Chemical Carcinogen Reference Standard Repository, Midwest Research Institute, Kansas City, Missouri.

55. D. C. Dong and M. A. Winnik, Can. J. Chem. 62, 2560 (1984).

56. K. W. Street, Jr., and W. E. Acree, Jr., Analyst 111, 1197 (1986).

57. S. A. Tucker, W. E. Acree, Jr., J. C. Fetzer, and J. Jacob, Polycyclic Aromat. Compds. 3, 1 (1992).

58. S. A. Tucker, V. L. Amszi, and W. E. Acree, Jr., J. Chem. Educ. 69, A8 (1992).

59. L. T. Scott, M. M. Hashemi, and M. S. Bratcher, J. Am. Chem. Soc. 114, 1902 (1992)

60. P. A. Peaden, M. L. Lee, Y. Hirata, and M. V. Novotny, Anal. Chem. 52, 2268 (1980)

61. K. W. Street, Jr., and W. E. Acree, Jr., J. Liq. Chromatogr. 9, 2799 (1986).

62. J. Michl and E. W. Thulstrup, Spectroscopy with Polarized Light (VCH Publishers, New York, 1986), Chap. 8.

63. W. E. Acree, Jr., S. A. Tucker, and J. C. Fetzer, Polycyclic Aromat. Compds. 1, 75 (1991). 